\title{
3 Research Square

\section{Validation of an Immune-related Gene Pair Index as a Prognostic Marker of Early-Stage Lung Squamous Cell Carcinoma}

\section{Zihao Wang}

Huazhong University of Science and Technology

\section{Xuan Xiang}

Huazhong University of Science and Technology

\section{Xiaoshan Wei}

Huazhong University of Science and Technology

\section{Linlin Ye}

Huazhong University of Science and Technology

\section{Yiran Niu}

Huazhong University of Science and Technology

\section{Wenbei Peng}

Huazhong University of Science and Technology

\section{Yu Li}

Huazhong University of Science and Technology

\section{Xu Wang}

Huazhong University of Science and Technology

\section{Siyu Zhang}

Huazhong University of Science and Technology

Qiong Zhou ( $\sim$ zhouqiongtj@126.com )

Huazhong University of Science and Technology https://orcid.org/0000-0002-3120-3017

\section{Research article}

Keywords: Lung squamous cell carcinoma, Immune-related genes, Prognosis

Posted Date: June 16th, 2020

DOI: https://doi.org/10.21203/rs.3.rs-32984/v1

License: (c) (1) This work is licensed under a Creative Commons Attribution 4.0 International License.

Read Full License 


\section{Abstract}

Background. Lung squamous cell carcinoma (LUSC) is one of the subtypes of non-small-cell lung cancer (NSCLC) and accounts for approximately 20 to $30 \%$ of all lung cancers.

Methods. In this study, we developed an immune-related gene pair index (IRGPI) for early-stage LUSC from 3 public LUSC data sets, including The Cancer Genome Atlas LUSC cohort and Gene Expression Omnibus data sets, and explored whether IRGPI could act as a prognostic marker to identify patients with early-stage LUSC at high risk.

Results. IRGPI was constructed by 68 gene pairs consisting of 123 unique immune-related genes from TCGA LUSC cohort. In the derivation cohort, the hazard of death among high-risk group was 10.51 times that of the low-risk group $(\mathrm{HR}, 10.51 ; 95 \% \mathrm{Cl}, 6.96-15.86 ; p<0.001)$. The hazard of death among the highrisk group was 2.26 times that of the low-risk group ( $\mathrm{HR}, 2.26 ; 95 \% \mathrm{Cl}, 1.2-4.25 ; \mathrm{p}=0.009)$ in the GSE37745 validation cohort and was 3.2 times that of low-risk group ( $H R, 3.2 ; 95 \% \mathrm{Cl}, 0.98-10.4 ; p=0.042)$ in the GSE41271 validation cohort. The infiltrations of $\mathrm{CD} 8^{+} \mathrm{T}$ cells and $\mathrm{T}$ follicular helper cells were lower in the high-risk group, as compared with the low-risk group in the TCGA cohort $(6.94 \%$ vs $9.63 \%, p=0.004$; $2.15 \%$ vs $3 \%, p=0.002$, respectively). The infiltrations of neutrophils, activated mast cells and monocytes were higher in the high-risk group, as compared with the low-risk group in the TCGA cohort $(1.63 \%$ vs $0.72 \%, p=0.001 ; 1.64 \%$ vs $1.02 \%, p=0.007 ; 0.57 \%$ vs $0.35 \%, p=0.041$, respectively).

Conclusions. IRGPI is a significant prognostic biomarker for predicting overall survival in early-stage LUSC patients.

\section{Introduction}

With the development of sequencing technology, more and more biomarkers were constructed for the diagnosis and prognosis of patients with lung cancer, including autoantibodies, complement fragments, miRNAs, circulating tumor DNA, DNA methylation, blood protein profiling, and other signatures. For example, Elena et al. reported a novel protein-based prognostic signature for risk stratification in patients with early lung adenocarcinoma[1], Ana et al. constructed an prognostic classifier based on mRNA, microRNA, and DNA methylation biomarkers to identify stage I lung adenocarcinoma patients at high risk of recurrence[2], and Daniel et al. described the diagnostic value of complement fragment C4d for patients with lung cancer[3].

As Guo et al. had reported, because of the instability of quantitative information of gene expression measurements under current technical conditions, it was more reasonable to construct quantitative transcriptional diagnostic signatures based on within-sample relative expression orderings of gene pairs[4]. Gene pairs signatures also demonstrated unique prognostic value in many cancers, such as nonsquamous non-small cell lung cancer[5], gastric cancer[6], and colorectal cancer[7]. 
Previous studies associated with prognosis of LUSC patients mainly concentrated on the alternative mRNA splicing signatures, IncRNA signatures, and other transcriptome signatures[8-13]. As the component of the tumor microenvironment, tumor-infiltrating lymphocytes (TIL) revealed promising value for providing prognostic biomarkers [14-16]. However, few studies focused on the immune-gene pairs signatures of LUSC patients.

In the present study, we firstly constructed an immune-related gene pair index (IRGPI) for early-stage LUSC and explored the prognostic value of IRGPI in the derivation and validation cohorts.

\section{Methods}

\section{Study Design and Data Process}

This retrospective study was conducted by using gene expression data from 3 public LUSC data sets, including The Cancer Genome Atlas (TCGA) LUSC cohort and Gene Expression Omnibus (GEO) data sets[17-19]. Only patients without neoadjuvant therapy, adjuvant chemotherapy or other immunemodulating therapy were included. LUSC RNA-Seq FPKM data and clinical information were downloaded from Genomic Data Commons Data Portal (GDC Data Portal) (https://portal.gdc.cancer.gov/). 385 earlystage LUSC patients with complete clinical information from TCGA were included as the derivation cohort. Two independent GEO data sets (GSE37745 and GSE41271) with clinical information were download from GEO (https://www.ncbi.nlm.nih.gov/geo/). The information of platforms was shown in sTable1 in the Supplement. For Affymetrix microarrays, gene expression profiles were normalized with the MAS5.0 method (affy, version 1.50.0)[20]. Model-Based Background Correction (MBCB) processed data were downloaded for Illumina datasets. A total of 54 and 47 early-stage LUSC patients from GSE37745 and GSE41271 datasets were enrolled as independent validation cohorts.

\section{Construction of IRGPI as a LUSC Specific Prognostic Biomarker}

The IRGPI was constructed according to a published article [5]. An immune-related genes (IRGs) list consisting of cytokines, cytokine receptors, and genes which related to variable immune cells function was constructed by using data downloaded from the ImmPort database (https://immport.niaid.nih.gov) [21]. Only IRGs which were detected by all platforms were included. To form immune-related gene pairs (IRGPs), the included genes were pairwise compared. For one gene pair, the IRGP score was 0 if the gene expression level of IRG 1 was more than that of IRG 2; otherwise, the IRGP score was 1. Immune-related gene pairs (IRGPs) with constant values ( 0 or 1 ) in each data set were excluded considering the probable biases. The log-rank test was used to select the IRGPs which were associated with overall survival in the derivation cohort. IRGP with a p-value of less than 0.01 was selected to construct the immune-related gene pair index (IRGPI). Cox proportional hazards regression model with the least absolute shrinkage and selection operator (LASSO) was used to select the appropriate IRGPs. The optimal model parameter $\lambda$ was evaluated by tenfold cross-validation at $1 \mathrm{SE}$ (standard error) as previously recommended. The timedependent receiver operating characteristic (ROC) curve was used to determine the optimal cutoff of 
IRGPI. The nearest neighbor estimation method was used to estimate the ROC curve. Patients were divided into low- and high-risk groups based on the cutoff of IRGPI.

\section{Validation of the Prognostic Value of IRGPI}

To assess the prognostic value of IRGPI, univariate Cox regression analysis was conducted in the derivation and validation cohorts. Age and tumor stage were coded as continuous variables. The tumor stage was coded as $I=1, \|=2$. The risk factors of gender and immune risk are male and high risk based on IRGPI. The prognostic accuracy of IRGPI was assessed by the time-dependent receiver operating characteristic (ROC) curve.

\section{Immune Cells Infiltration of Low- and High- Risk Groups}

Tumor-infiltrating leukocytes (TILs) are important components of the tumor microenvironment (TME). The infiltrations of immune cells were assessed by CIBERSORT[22]. CIBERSORT is a computational method that can quantify immune cell fractions by using gene expression profiles (GEPs) from bulk tumor tissue. LUSC RNA-Seq gene expression profiles were downloaded from TCGA and transformed into the appropriate form according to the requirement. A total of 22 immune cell fractions were evaluated via the CIBERSORT website (https://cibersort.stanford.edu/). Wilcoxon rank-sum test was used to assess the difference of immune infiltrations between low- and high-risk groups.

\section{Functional Annotation of Immune-related Genes}

IRGPI was constructed by 68 IRGPs (123 unique genes). The functional annotation of these genes was conducted using clusterProfiler (version 3.0.4)[23]. The cellular component, molecular function and biological process of gene ontology were shown in sFig1 in the Supplement.

\section{Statistical analysis}

All statistical analyses were performed using R version 3.6.1(https://www.r-project.org/). The log-rank test was used to select Immune-related Gene Pairs (IRGPs) associated with overall survival in the derivation cohort. LASSO Cox proportional hazards regression model was used to select ideal prognostic IRGPs for the construction of IRGPI by gImnet (version 2.0-18)[24]. The time-dependent receiver operating characteristic (ROC) curve was applied to determine the optimal cutoff of IRGPI by survivalROC (version 1.0.3)[25]. Derivation cohort and validation cohort were separated into high-risk and low-risk groups by the same cutoff. The discrimination of the model was evaluated by Area Under the Curve (AUC) of the ROC curve. The Kaplan-Meier method was used to estimate survival curves. Multivariate Cox proportional hazards regression model was performed with variates significantly associated with overall survival in the univariate analysis by survival (version 2.44-1.1) packages. Unless specified otherwise, $\mathrm{p}$ value $<0.05$ was considered statistically significant.

\section{Results}




\section{Construction and Validation of the IRGPI}

Table 1 summarized the clinical characteristics of patients enrolled in this study. 1179 IRGs were detected by the platforms mentioned in the sTable1 in the Supplement. 694431 IRGPs were constructed and $97.4 \%$ of them were excluded. The log-rank test was used to assess the association between the remaining 18396 IRGPs and the overall survival of patients in the derivation cohort. 401 IRGPs with a p-value of less than 0.01 were selected to fit a Cox proportional hazards regression model with the least absolute shrinkage and selection operator (LASSO) (Fig 1.a, b). The final 68 IRGPs and LASSO coefficients were shown in sTable2 in the Supplement. 68 IRGPs (123 unique immune-related genes) were used to construct IRGPI via L1-penalized Cox proportional hazards regression in the derivation data set. Based on the time-dependent ROC curve analysis, the optional cutoff for IRGPI was 1.32 (sFig2 in the Supplement). Survival curves of low- and high-risk groups were estimated by using the Kaplan-Meier method and were compared by using the log-rank test in the derivation and validation cohorts (Fig 1.c and e). In the TCGA derivation cohort, the AUC of time-dependent ROC curves at 1,3 and 5 years was $0.854,0.953$ and 0.944 , respectively (Fig 1.d). In the GSE37745 validation cohort, the AUC of time-dependent ROC curves at 1, 3 and 5 years was $0.764,0.728$ and 0.710 , respectively (Fig 1.f).

\section{Validation of IRGP as a prognostic factor of Early-stage LUSC}

Hazard ratios between high-risk and low-risk groups based on IRGPI were shown in the forest plot (Fig 2). The IRGPI stratified patients with early-stage (stage I and II) LUSC into different prognostic groups. In early-stage (stage I and II) LUSC, the hazard of death among high-risk group was 10.51 times that of lowrisk group ( $\mathrm{HR}, 10.51 ; 95 \% \mathrm{Cl}, 6.96-15.86 ; \mathrm{p}<0.001)$ in the TCGA derivation cohort. In early-stage (stage I and II) LUSC, the hazard of death among high-risk group was 2.26 times that of low-risk group (HR, 2.26; $95 \% \mathrm{Cl}, 1.2-4.25 ; \mathrm{p}=0.009)$ in the GSE37745 validation cohort and was 3.2 times that of low-risk group (HR, 3.2; $95 \% \mathrm{Cl}, 0.98-10.4 ; \mathrm{p}=0.042)$ in the GSE41271 validation cohort. In stage I LUSC, the hazard of death among high-risk group was 9.77 times that of low-risk group (HR, 9.77; 95\% $\mathrm{Cl}, 5.86-16.3 ; p<0.001)$ in the derivation cohort and was 2.33 times that of low-risk group $(\mathrm{HR}=2.33,95 \% \mathrm{Cl}, 1.11-4.88 ; \mathrm{p}=0.021)$ in the GSE37745 validation cohort. In stage II LUSC, the IRGPI remained prognostic accuracy for the derivation cohort $(H R=11.39 ; 95 \% \mathrm{Cl}, 5.66-22.9 ; p<0.001)$ and $G S E 41271$ validation cohort $(H R=9.8 ; 95 \%$ $\mathrm{Cl}, 0.98-98.2 ; \mathrm{p}=0.02)$. Table 2 demonstrated the univariate and multivariate analyses of IRGPI and clinical characteristics. In the GSE37745 validation cohort, IRGPI (HR, 2.95; 95\% Cl, 1.5-5.79; $\mathrm{p}=0.002)$ and old age $(\mathrm{HR}, 1.05 ; 95 \% \mathrm{Cl}, 1.01-1.1$; per year increase; $p=0.026)$ were independent risk factors for poor prognosis.

\section{Immune Infiltration related to IRGPI}

Gene ontology (GO) of the unique 123 LUSC IRGs was shown in the sFig1 in the Supplement. Most of the biological processes were cytokine biosynthetic, secreted and metabolic processes. The molecular function of GO concentrated on cytokine receptor binding, receptor-ligand activity, receptor regulator activity, etc. In the early-stage LUSC TCGA cohort, the percentages of 22 immune cells infiltration were shown in Fig 3.a and Fig 3.b. Patients in high-risk group had higher proportions of neutrophils, monocytes 
and activated mast cells infiltrations in their tumors ( $1.63 \%$ vs $0.72 \%, p=0.001 ; 0.57 \%$ vs $0.35 \%, p=0.041$; $1.64 \%$ vs $1.03 \%, p=0.007$, respectively). But the infiltrations of CD $8+T$ cells and $T$ follicular helper cells were lower in the high-risk group, as compared with the low-risk group (6.94\% vs $9.63 \%, p=0.004 ; 2.15 \%$ vs $3 \%, p=0.002$, respectively) (Fig 3.c).

\section{Comparison of biomarkers for LUSC}

We summarized current biomarkers for LUSC and compared the biomarkers in Table 3. Li et al. constructed a model with 6 IncRNAs from TCGA LUSC cohort and the area under the curve (AUC) of the 6IncRNA signature associated with 3-year survival was 0.672 in the training cohort[11]. Hu et al. constructed a 3-IncRNA signature for LUSC and the AUC of this model associated with 3-year survival was 0.629 in the training cohort[12]. Qi et al. identified 12 miRNAs closely related to the overall survival of patients with LUSC[13]. Yang et al. identified the diagnostic role of miRNA-486-5p in TCGA LUSC cohort[26]. Li et al. found that 60 genes were statistically related to the overall survival rate in LUSC patients[10]. Shi et al. identified 6 methylation biomarkers for LUSC diagnosis[27]. Li et al. created prognostic predictors based on alternative splicing events for NSCLC patients and the AUC for prognostic predictor was over 0.8 in TCGA LUSC cohort[9]. Choi et al. found that MLL2 mutations predicted poor prognosis in both TP53 mutant and wild-type LUSC[8]. Gao et al. identified a prognostic model contained 5 genes and the AUC of the model for predicting the survival at 1,3 , and 5 years was $0.692,0.722$, and 0.651 , respectively[28]. For TCGA derivation cohort, the AUC of IRGPI in at 1, 3 and 5 years was 0.854 , 0.953 and 0.944 , respectively. For GSE37745 validation cohort, the AUC of IRGPI at 1, 3 and 5 years was $0.764,0.728$ and 0.710 , respectively.

\section{Discussion}

Gene expression profiles had shown enormous potentials in predicting the survival of patients. Many biomarkers from gene signatures were constructed for the evaluation of prognosis in patients with nonsquamous NSCLC, such as CCP score [29] and quantitative-PCR-based assay [30]. The cell-cycle progression genes (CCP score) were calculated from the normalized signatures of 31 cell-cycle genes and could identify patient groups of reduced or increased risk of death after surgical resection. The quantitative-PCR-based assay could identify patients with early-stage non-squamous NSCLC at high risk for mortality after surgical resection.

Current prognostic biomarkers for lung squamous cell carcinoma (LUSC) included prognostic alternative mRNA splicing signature, IncRNA signatures, and microRNA signatures[9, 11-13]. However, these studies $[9,11-13]$ only included LUSC data from TCGA database. In this study, we built IRGPI based on 68 immune-related gene pairs from TCGA LUSC database and explored the prognostic value of IRGPI in two independent GEO data sets. By combining multi-gene expression, it was possible to construct robust gene expression-based biomarkers to stratify patients with LUSC into low-risk and high-risk groups, even if gene expression profiles were from different sequencing platforms. Results showed that IRGPI could stratify early-stage LUSC patients into subgroups with different survival outcomes. Besides, we found 
that IRGPI and old age were independent risk factors for poor prognosis in the GSE37745 validation cohort.

The immunotherapy for NSCLC could be improved by prognostic or predictive biomarkers related to the tumor immune microenvironment. According to previous study, patients with lower levels of tumorinfiltrating effector $T$ cells demonstrated a relatively worse prognosis [31]. In this study, we found a higher infiltration of neutrophils and a lower infiltration of $\mathrm{CD} 8^{+} \mathrm{T}$ cells in the high-risk group based on IRGPI in the TCGA cohort. Patients in the high-risk group had lower levels of tumor-infiltrating $\mathrm{CD} 8^{+} \mathrm{T}$ cells and displayed relatively poor prognosis. Dysregulation of tumor-infiltrating lymphocytes might explain the differences in the overall survival between low- and high-risk groups based on IRGPI.

To construct a robust signature, several LUSC data sets were included in the study. The expression levels of genes in each sample were compared in pairs to generate IRGPI. This gene pair-based approach was based on the relative gene expression of each sample and did not need normalization. There were also some limitations in our study. Although batch effects were reduced by the pairwise comparison of immune-related genes, some of them were unavoidable due to the combination of different platforms [32]. Meanwhile, sampling bias caused by intratumor genetic heterogeneity was inevitable for gene expression-based IRGPI.

\section{Conclusions}

The immune-related gene pair index (IRGPI) is a prospective prognostic biomarker for LUSC, especially for early-stage LUSC. Further studies are still needed to validate its accuracy for assessing prognosis and to explore its clinical utility in the individualized management of LUSC.

\section{Declarations}

\section{Acknowledgements}

We would like to thank GuoZiXueShengXin for the bioinformatic skills.

\section{Authors' contributions}

Study design: WZH, WXS, PWB, YLL, NYR. Data acquisition: LY, ZSY, XX. Data analysis: WZH. Drafting of the manuscript: WZH, WXS. Revision of the manuscript: ZQ. All authors contributed significantly to this work and agreed to be accountable for the work. All authors read and approved the final manuscript.

\section{Funding}

This work was supported in part by grants from National Natural Science Foundation of China (No. 81973990, No. 81900096, No.81770090).

\section{Availability of data and materials}


All datasets were adopted in this study are available in the GEO (https://www.ncbi.nlm.nih.gov/geo/) and TCGA (https://portal.gdc.cancer.gov/) database.

\section{Ethics approval and consent to participate}

Appropriate Ethics Committees of Tongji Medical College approved this study to be exempt research.

\section{Consent for publication}

Not applicable.

\section{Competing interests}

The authors declare that they have no competing interests.

\section{References}

1. Martínez-Terroba E, Behrens C, de Miguel FJ, Agorreta J, Monsó E, Millares L, Sainz C, Mesa-Guzman M, Pérez-Gracia JL, Lozano MD et al: A novel protein-based prognostic signature improves risk stratification to guide clinical management in early-stage lung adenocarcinoma patients. The Journal of pathology 2018, 245(4):421-432.

2. Robles Al, Arai E, Mathé EA, Okayama H, Schetter AJ, Brown D, Petersen D, Bowman ED, Noro R, Welsh JA et al: An Integrated Prognostic Classifier for Stage I Lung Adenocarcinoma Based on mRNA, microRNA, and DNA Methylation Biomarkers. Journal of thoracic oncology : official publication of the International Association for the Study of Lung Cancer 2015, 10 (7):1037-1048.

3. Ajona D, Pajares MJ, Corrales L, Perez-Gracia JL, Agorreta J, Lozano MD, Torre W, Massion PP, deTorres JP, Jantus-Lewintre $\mathrm{E}$ et al: Investigation of complement activation product $\mathrm{c} 4 \mathrm{~d}$ as a diagnostic and prognostic biomarker for lung cancer. Journal of the National Cancer Institute 2013, 105(18):1385-1393.

4. Guan Q, Yan H, Chen Y, Zheng B, Cai H, He J, Song K, Guo Y, Ao L, Liu H et al: Quantitative or qualitative transcriptional diagnostic signatures? A case study for colorectal cancer. BMC genomics 2018, 19(1):99.

5. Li B, Cui Y, Diehn M, Li R: Development and Validation of an Individualized Immune Prognostic Signature in Early-Stage Nonsquamous Non-Small Cell Lung Cancer. JAMA oncology 2017, 3(11):1529-1537.

6. Peng PL, Zhou XY, Yi GD, Chen PF, Wang F, Dong WG: Identification of a novel gene pairs signature in the prognosis of gastric cancer. Cancer medicine 2018, 7(2):344-350.

7. Wu J, Zhao Y, Zhang J, Wu Q, Wang W: Development and validation of an immune-related gene pairs signature in colorectal cancer. Oncoimmunology 2019, 8(7):1596715.

8. Choi M, Kadara H, Zhang J, Parra ER, Rodriguez-Canales J, Gaffney SG, Zhao Z, Behrens C, Fujimoto $\mathrm{J}$, Chow $\mathrm{C}$ et al: Mutation profiles in early-stage lung squamous cell carcinoma with clinical follow-up 
and correlation with markers of immune function. Annals of oncology : official journal of the European Society for Medical Oncology 2017, 28(1):83-89.

9. Li Y, Sun N, Lu Z, Sun S, Huang J, Chen Z, He J: Prognostic alternative mRNA splicing signature in non-small cell lung cancer. Cancer letters 2017, 393:40-51.

10. Li Y, Gu J, Xu F, Zhu Q, Ge D, Lu C: Transcriptomic and functional network features of lung squamous cell carcinoma through integrative analysis of GEO and TCGA data. Scientific reports 2018, 8(1):15834.

11. Li R, Yang YE, Jin J, Zhang MY, Liu X, Liu XX, Yin YH, Qu YQ: Identification of IncRNA biomarkers in lung squamous cell carcinoma using comprehensive analysis of IncRNA mediated ceRNA network. Artificial cells, nanomedicine, and biotechnology 2019, 47(1):3246-3258.

12. Hu J, Xu L, Shou T, Chen Q: Systematic analysis identifies three-IncRNA signature as a potentially prognostic biomarker for lung squamous cell carcinoma using bioinformatics strategy. Translational lung cancer research 2019, 8(5):614-635.

13. Qi L, Gao C, Feng F, Zhang T, Yao Y, Wang X, Liu C, Li J, Li J, Sun C: MicroRNAs associated with lung squamous cell carcinoma: New prognostic biomarkers and therapeutic targets. Journal of cellular biochemistry 2019, 120(11):18956-18966.

14. Coussens LM, Werb Z: Inflammation and cancer. Nature 2002, 420(6917):860-867.

15. Zhang L, Conejo-Garcia JR, Katsaros D, Gimotty PA, Massobrio M, Regnani G, Makrigiannakis A, Gray $\mathrm{H}$, Schlienger $\mathrm{K}$, Liebman MN et al: Intratumoral T cells, recurrence, and survival in epithelial ovarian cancer. The New England journal of medicine 2003, 348(3):203-213.

16. Angell $\mathrm{H}$, Galon J: From the immune contexture to the Immunoscore: the role of prognostic and predictive immune markers in cancer. Current opinion in immunology 2013, 25(2):261-267.

17. Comprehensive genomic characterization of squamous cell lung cancers. Nature 2012, 489(7417):519-525.

18. Botling J, Edlund K, Lohr M, Hellwig B, Holmberg L, Lambe M, Berglund A, Ekman S, Bergqvist M, Ponten $\mathrm{F}$ et al: Biomarker discovery in non-small cell lung cancer: integrating gene expression profiling, meta-analysis, and tissue microarray validation. Clinical cancer research : an official journal of the American Association for Cancer Research 2013, 19(1):194-204.

19. Sato M, Larsen JE, Lee W, Sun H, Shames DS, Dalvi MP, Ramirez RD, Tang H, DiMaio JM, Gao B et al: Human lung epithelial cells progressed to malignancy through specific oncogenic manipulations. Molecular cancer research : MCR 2013, 11(6):638-650.

20. Gautier L, Cope L, Bolstad BM, Irizarry RA: affy--analysis of Affymetrix GeneChip data at the probe level. Bioinformatics (Oxford, England) 2004, 20(3):307-315.

21. Bhattacharya S, Andorf S, Gomes L, Dunn P, Schaefer H, Pontius J, Berger P, Desborough V, Smith T, Campbell $\mathrm{J}$ et al: ImmPort: disseminating data to the public for the future of immunology. Immunologic research 2014, 58(2-3):234-239.

22. Chen B, Khodadoust MS, Liu CL, Newman AM, Alizadeh AA: Profiling Tumor Infiltrating Immune Cells with CIBERSORT. Methods in molecular biology (Clifton, NJ) 2018, 1711:243-259. 
23. Yu G, Wang LG, Han Y, He QY: clusterProfiler: an R package for comparing biological themes among gene clusters. Omics : a journal of integrative biology 2012, 16(5):284-287.

24. Simon N, Friedman J, Hastie T, Tibshirani R: Regularization Paths for Cox's Proportional Hazards Model via Coordinate Descent. Journal of statistical software 2011, 39(5):1-13.

25. Saha-Chaudhuri P, Heagerty PJ: Non-parametric estimation of a time-dependent predictive accuracy curve. Biostatistics (Oxford, England) 2013, 14(1):42-59.

26. Yang S, Sui J, Liu T, Wu W, Xu S, Yin L, Pu Y, Zhang X, Zhang Y, Shen B et al: Expression of miR-486$5 p$ and its significance in lung squamous cell carcinoma. Journal of cellular biochemistry 2019, 120(8):13912-13923.

27. Shi YX, Wang Y, Li X, Zhang W, Zhou HH, Yin JY, Liu ZQ: Genome-wide DNA methylation profiling reveals novel epigenetic signatures in squamous cell lung cancer. BMC genomics 2017, 18(1):901.

28. Gao M, Kong W, Huang Z, Xie Z: Identification of Key Genes Related to Lung Squamous Cell Carcinoma Using Bioinformatics Analysis. International journal of molecular sciences 2020, 21(8).

29. Wistuba, II, Behrens C, Lombardi F, Wagner S, Fujimoto J, Raso MG, Spaggiari L, Galetta D, Riley R, Hughes $\mathrm{E}$ et al: Validation of a proliferation-based expression signature as prognostic marker in early stage lung adenocarcinoma. Clinical cancer research : an official journal of the American Association for Cancer Research 2013, 19(22):6261-6271.

30. Kratz JR, He J, Van Den Eeden SK, Zhu ZH, Gao W, Pham PT, Mulvihill MS, Ziaei F, Zhang H, Su B et al: A practical molecular assay to predict survival in resected non-squamous, non-small-cell lung cancer: development and international validation studies. Lancet (London, England) 2012, 379(9818):823-832.

31. Schalper KA, Brown J, Carvajal-Hausdorf D, McLaughlin J, Velcheti V, Syrigos KN, Herbst RS, Rimm DL: Objective measurement and clinical significance of TILs in non-small cell lung cancer. Journal of the National Cancer Institute 2015, 107(3).

32. Leek JT, Scharpf RB, Bravo HC, Simcha D, Langmead B, Johnson WE, Geman D, Baggerly K, Irizarry RA: Tackling the widespread and critical impact of batch effects in high-throughput data. Nature reviews Genetics 2010, 11(10):733-739.

\section{Tables}

Table 1. Clinical characteristics of patients enrolled in this study 


\begin{tabular}{llll} 
Variable & Derivation & Validation & \\
\cline { 2 - 4 } & TCGA & GSE37745 & GSE41271 \\
\hline No. of patients & 385 & 54 & 47 \\
\hline Median ages in years(range) & $69(39-90)$ & $67.5(40-84)$ & $65.5(45-86)$ \\
\hline Gender & & & \\
\hline Female(\%) & $102(26.5)$ & $17(31.5)$ & $20(42.6)$ \\
\hline Male(\%) & $283(73.5)$ & $37(68.5)$ & $27(57.4)$ \\
\hline Stage & & & \\
\hline I(\%) & $230(59.7)$ & $4074.1)$ & $30(63.8)$ \\
\hline II(\%) & $155(40.3)$ & $14(25.9)$ & $17(36.2)$ \\
\hline Median Follow-up in Days & 741 & 1257 & 1310 \\
\hline No. of death(\%) & $152(39.5)$ & $41(75.9)$ & $16(34)$
\end{tabular}

Table 2. Univariate and multivariate analyses of IRGPI and clinical characteristics in the derivation and validation data sets. 
Cohort

characteristics Univariate Multivariate

HR $95 \% \mathrm{Cl}$ P.Value HR $95 \% \mathrm{Cl}$ P.Value

Early stage
(stage I and
stage II)

\begin{tabular}{|c|c|c|c|c|c|c|c|}
\hline \multirow[t]{4}{*}{ Derivation } & risk & 10.5 & $\begin{array}{l}6.96- \\
15.9\end{array}$ & $<0.001$ & - & - & - \\
\hline & gender & 1.18 & $\begin{array}{l}0.81- \\
1.73\end{array}$ & 0.391 & - & - & - \\
\hline & age & 1.02 & $1-1.04$ & 0.19 & - & - & - \\
\hline & stage & 1.16 & $\begin{array}{l}0.84- \\
1.61\end{array}$ & 0.375 & - & - & - \\
\hline \multirow[t]{4}{*}{ GSE37745 } & risk & 2.26 & $\begin{array}{l}1.2- \\
4.25\end{array}$ & 0.009 & 2.95 & $\begin{array}{l}1.5- \\
5.79\end{array}$ & 0.002 \\
\hline & gender & 0.83 & $\begin{array}{l}0.43- \\
1.63\end{array}$ & 0.603 & - & - & - \\
\hline & age & 1.03 & $\begin{array}{l}0.99- \\
1.07\end{array}$ & $<0.001$ & 1.05 & $\begin{array}{l}1.01- \\
1.1\end{array}$ & 0.026 \\
\hline & stage & 0.98 & $\begin{array}{l}0.48- \\
2.01\end{array}$ & 0.959 & - & - & - \\
\hline \multirow[t]{4}{*}{ GSE41271 } & risk & 3.2 & $\begin{array}{l}0.98^{-} \\
10.4\end{array}$ & 0.042 & 3.18 & $\begin{array}{l}0.98- \\
10.34\end{array}$ & 0.054 \\
\hline & gender & 0.84 & $\begin{array}{l}0.31- \\
2.27\end{array}$ & 0.73 & - & - & - \\
\hline & age & 1.01 & $\begin{array}{l}0.95- \\
1.07\end{array}$ & $<0.001$ & 1.01 & $\begin{array}{l}0.95- \\
1.07\end{array}$ & 0.794 \\
\hline & stage & 1.07 & $\begin{array}{l}0.37- \\
3.09\end{array}$ & 0.907 & - & - & - \\
\hline
\end{tabular}

Table 3. Comparison of different biomarkers for LUSC. 


\begin{tabular}{|c|c|c|c|c|c|}
\hline Biomarkers & Signature & Datasets & $\begin{array}{l}\text { AUC of } \\
\text { derivation } \\
\text { cohort }\end{array}$ & $\begin{array}{l}\text { AUC of validation } \\
\text { cohort }\end{array}$ & PMID \\
\hline $\begin{array}{l}\text { A model with } 6 \\
\text { IncRNAs }\end{array}$ & LncRNA & TCGA & 0.629 & - & 31364871 \\
\hline $\begin{array}{l}\text { A model with } 3 \\
\text { IncRNAs }\end{array}$ & LncRNA & TCGA & 0.672 & - & 31737498 \\
\hline 12 microRNAs & MicroRNA & TCGA & - & - & 31241205 \\
\hline MiR-486-5p & MicroRNA & TCGA & 0.908 & - & 30963622 \\
\hline 60 Genes & Gene & $\begin{array}{l}\text { TCGA } \\
\text { GEO }\end{array}$ & - & - & 30367091 \\
\hline $\begin{array}{l}\text { Methylation } \\
\text { biomarkers }\end{array}$ & Gene & TCGA & - & $>0.9$ & 29169318 \\
\hline $\begin{array}{l}\text { Alternative mRNA } \\
\text { splicing events }\end{array}$ & mRNA & TCGA & $>0.8$ & - & 28223168 \\
\hline Mutated genes & Mutation & - & - & - & 28177435 \\
\hline $\begin{array}{l}\text { A model contained } 5 \\
\text { genes }\end{array}$ & Gene & TCGA & - & $0.692 / 0.722 / 0.651$ & 32340320 \\
\hline
\end{tabular}

\section{Figures}


a

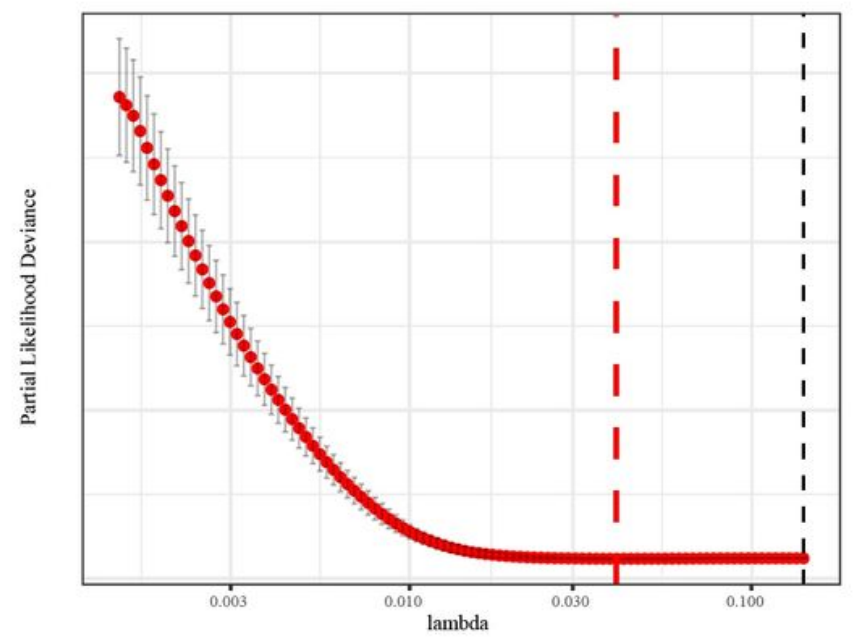

C

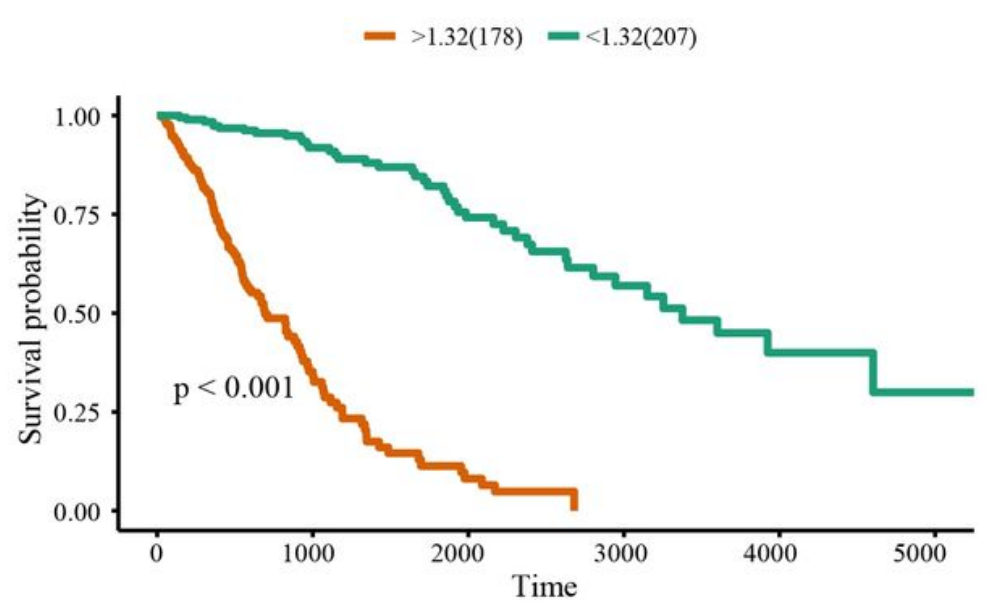

e

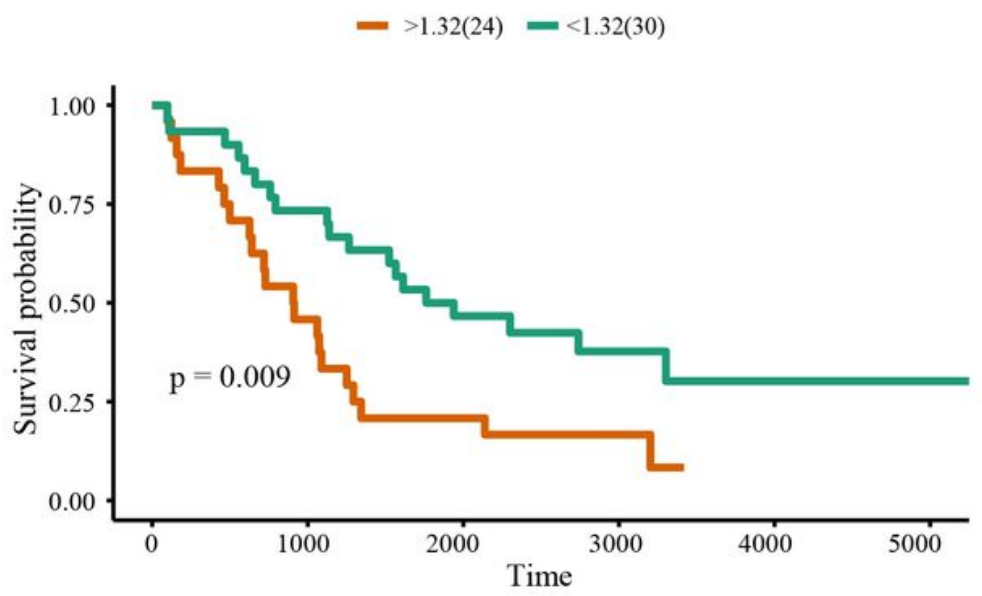

b

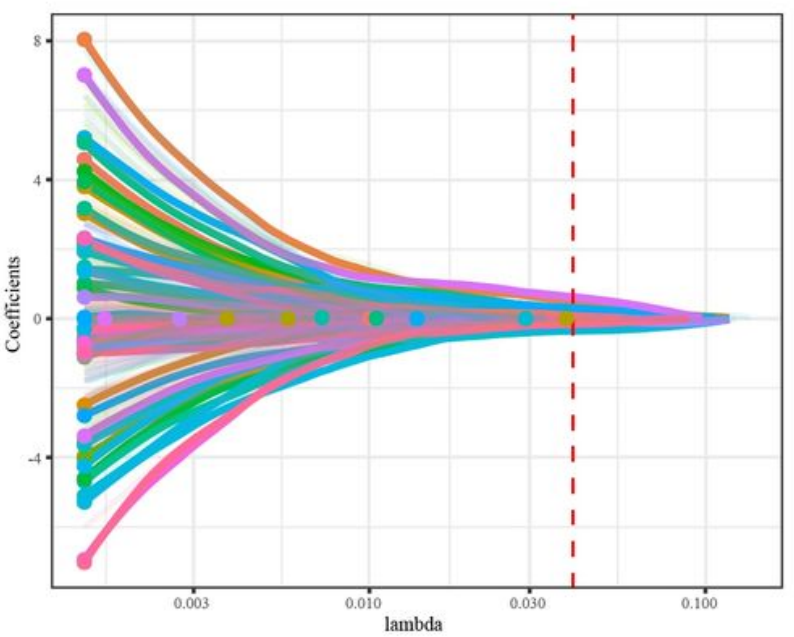

d

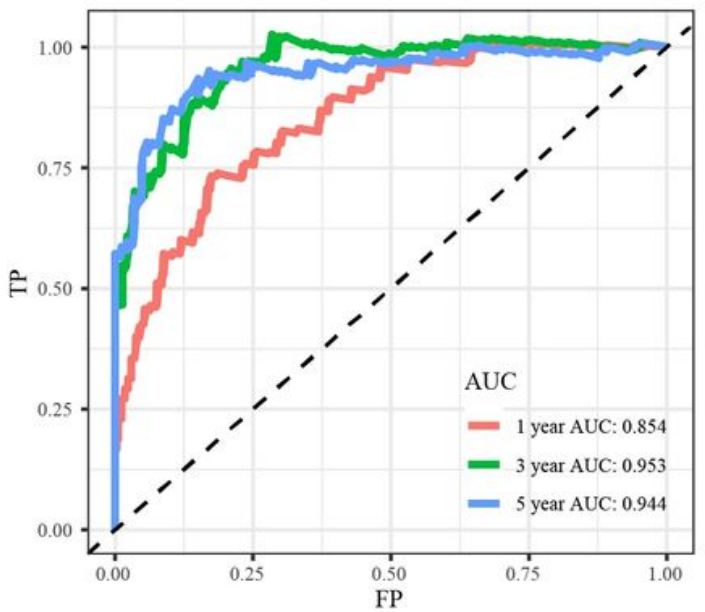

f

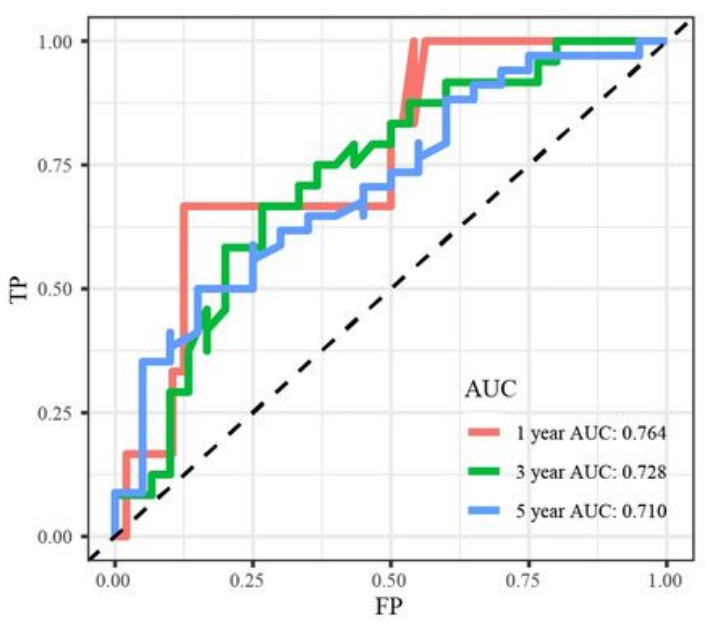

Figure 1

Patients were divided into low- and high-risk groups by IRGPI. (a, b) The penalty parameter was estimated by 10 -fold cross-validation in the derivation data set at 1 SE beyond the minimum partial likelihood deviance. (c, e) The survival curves of low- and high-risk groups in the TCGA derivation and GSE37745 validation cohort. ( $d, f)$ The ROC curves and AUC of low- and high-risk groups in the TCGA derivation and GSE37745 validation cohort. 


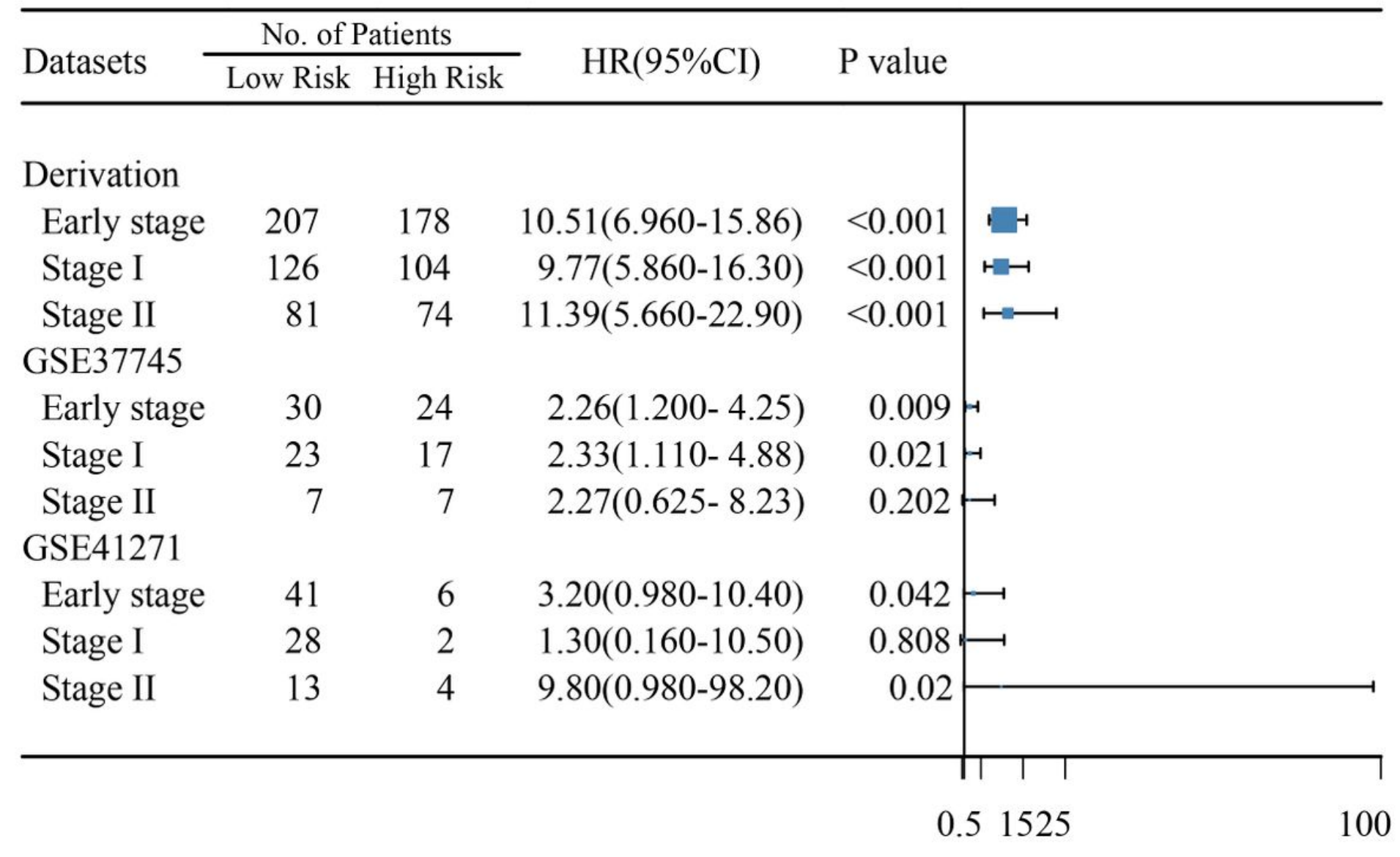

Figure 2

HRs between IRGPI high- and low-risk groups were evaluated using univariate Cox proportional hazards regression. The length of the horizontal line corresponds to the confidence interval, and the size of the HR data marker is proportional to the number of patients. The vertical line indicates that HR is equal to 1 . 


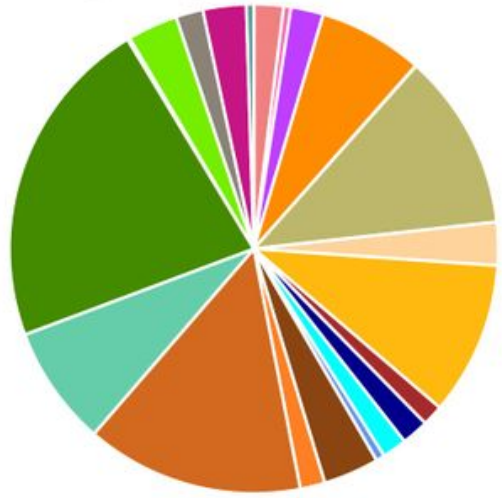

Mean infiltrations of 22 immune cells

b Low-risk group based on IRGPI

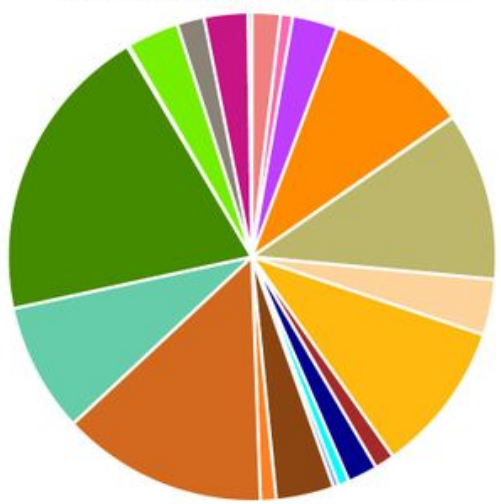

Mean infiltrations of 22 immune cells
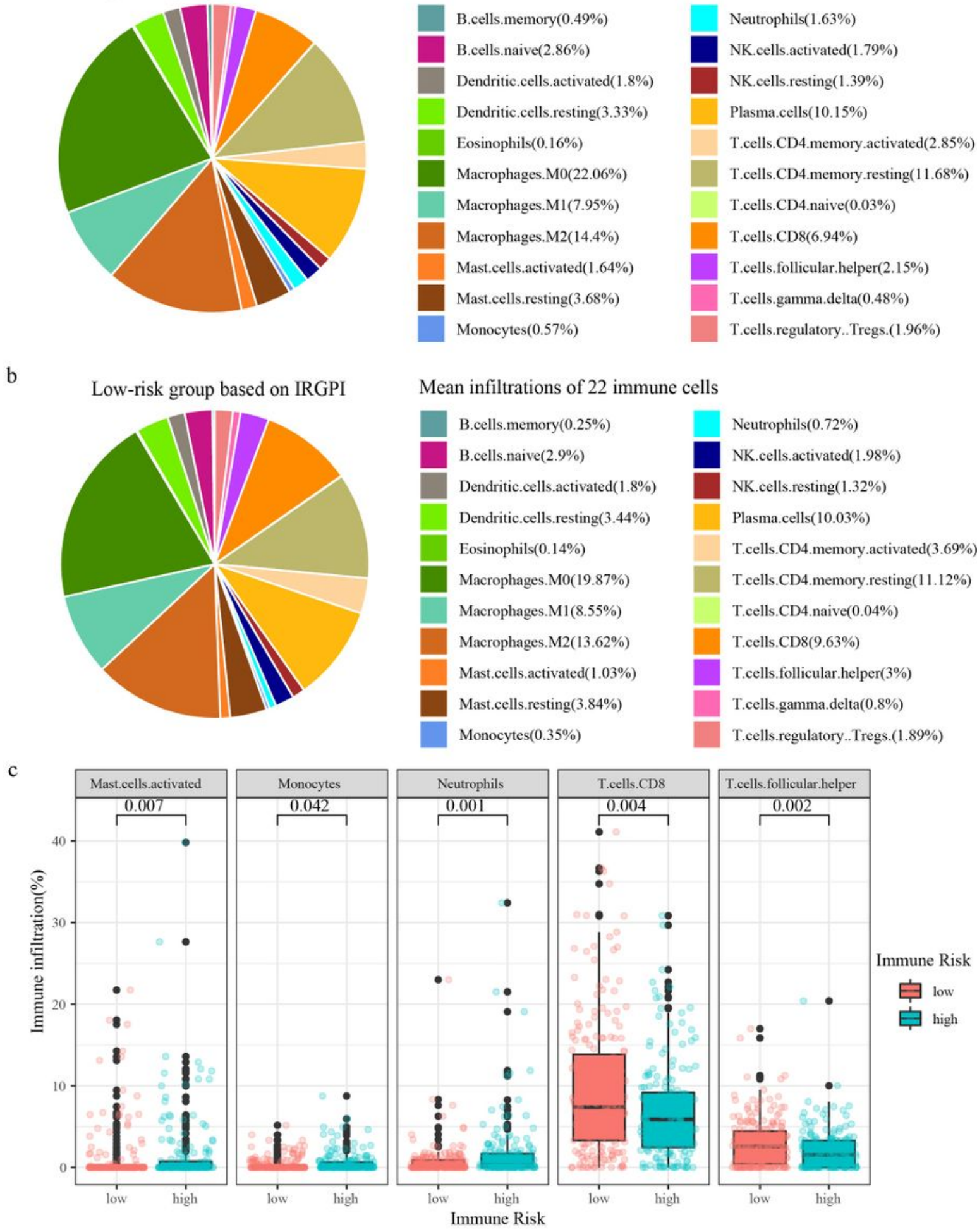

\section{Figure 3}

Patients were divided into low- and high-risk groups by IRGPI. a The immune infiltrations of the high-risk group based on IRGPI. b The immune infiltrations of the low-risk group based on IRGPI. c The immune infiltrations of groups were compared by Wilcoxon rank-sum test.

\section{Supplementary Files}


This is a list of supplementary files associated with this preprint. Click to download.

- Supplement.pdf 